reported that as a result of the simulation, they felt their approach to safeguarding had changed and they now felt "more confident to ask the difficult questions", "protect the children they were seeing" and "set a good example to their junior colleagues".

Conclusion We successfully ran a Safeguarding simulation to help prepare junior trainees for their role in the initial management of a child with safeguarding concerns. It is a scalable learning exercise, which is low tech and easily reproducible in local safeguarding courses. We would recommend the use of simulation for this purpose.

\section{G216 A META-ANALYSIS OF "WII THERAPY" IN CHILDREN WITH CEREBRAL PALSY}

doi:10.1136/archdischild-2013-304107.228

W Farr, I Male. Mid Sussex Child Development Centre, Sussex Community Trust, Haywards Heath, UK

Aims There has been growing interest in the therapeutic potential of the Nintendo Wii and Wii Fit (Wii) as Virtual Rehabilitation Therapy tools in conditions affecting motor function such as stroke, Parkinsons disease, ataxia, DCD, and cerebral palsy (CP). Although one case report showed significant gains in an adolescent with diplegic cerebral palsy, there was previously only a limited evidence base on use in children. This meta-analysis focused on RCTs in CP and aimed to calculate effect sizes to see if further trials are warranted.

Methods Eleven databases were used to find Wii RCTs. Studies were filtered by a focus on i) Cerebral Palsy ii) published 2006 - 2012 covering the availability of the Wii and iii) discounting broader virtual therapy. Effect size was calculated using Cohen's d. Effect size below 0.3 was classified as small, 0.3 to 0.8 medium and $0.8+$ as large.

Results With the heterogeneous nature of CP a number of different tools and outcomes were measured in the different studies, and numbers were generally too small to give statistically significant results. However, two studies meeting criteria $(\mathrm{N}=29,14)$ showed effect sizes of 0.74 and 0.60 for balance. One study $(n=6)$ showed effect size 0.30 for motor function (GMFM). Other studies measured outcomes such as manual dexterity, bone mineral density or energy expenditure with variable results.

Conclusion The two studies assessing balance showed moderate effect sizes. This is in agreement with research looking at improvements in balance in other motor disorders including our own study in children with DCD, acquired brain injury, spinocerebellar ataxia, adults with stroke, and case reports in children with CP. Lack of uniformity across research hinders understanding of whether the Wii is effective paediatric intervention. Studies are predominantly pilot phase, lack agreement over measurement tools, use small sample sizes, and few had calculated power and sample size. Studies also suffer from selection bias due to the motivational aspects of the Wii. Nevertheless, results suggest there may well be significant therapeutic gain in motor function for children with cerebral palsy, warranting larger-scale and more definitive studies.

\section{G217(P) VALIDATION OF A PROPOSED CLINICAL TOOL TO ESTIMATE THE PROBABILITY OF ABUSIVE HEAD TRAUMA IN CHILDREN AGED LESS THAN THREE YEARS}

doi:10.1136/archdischild-2013-304107.229

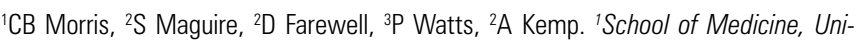
versity Hospital of Wales, Cardiff, UK' ${ }^{2}$ Child Protection, University Hospital of Wales, Cardiff, UK; ${ }^{3}$ Paediatric Ophthalmology, University Hospital of Wales, Cardiff, UK

Background Abusive head trauma (AHT) is the most common cause of death and disability in abused children, and presents significant diagnostic challenges. Previous research identified six individual features (retinal haemorrhage, rib and long bone fractures, facial bruising, apnoea and seizures) associated with AHT to create a statistical model to determine the probability of AHT based upon different combinations of these features in a child with intracranial injury.

Aims The primary aim was to independently validate the statistical model on a novel dataset. The secondary aim was to look for association between AHT and the original six features, and further features not included in the original model, to suggest areas for refinement.

Methods Retrospective, notes-based review of 44 cases of children aged less than 36 months admitted with intracranial head injury $(20$ AHT), identified at neuroimaging (01/01/2007-31/02/2012). Sensitivity, Specificity, Positive Predictive Value (PPV) and Negative Predictive Value (NPV) were calculated to determine the model's accuracy. Fisher's Exact Test and logistic regression were used to test for association between individual features and AHT.

\section{Results}

Abstract G217(P) Table 1

\begin{tabular}{ll}
\hline & Values \\
\hline Sensitivity & $84.2-87.5 \%$ \\
Specificity & $29.2-86.4 \%$ \\
NPV & $70-76.2 \%$ \\
PPV & $51.4-84.2 \%$ \\
\hline
\end{tabular}

Significant association was found between AHT and retinal haemorrhage $(p<0.001)$, seizures $(p<0.02)$. Strong but not significant association was found between AHT and apnoea $(p<0.08)$, and between non-AHT and skull fracture $(p<0.25)$. Subdural haemorrhage, not included in the original model, was significantly associated with AHT ( $p<0.04)$ On sub-analysis of retinal features, too numerous to count retinal haemorrhage was significantly associated with AHT $(p<0.04)$. Retinal haemorrhages were more likely to be multi-layered and bilateral in AHT cases.

Conclusions When tested on this dataset the model had similar sensitivity and specificity to the original study, although imputing data caused variation. Type of intracranial injury and specific retinal features were identified as areas for refinement. The high sensitivity suggests that the tool has the potential to identify cases of suspected AHT that Warrant further detailed assessment, and could be useful for clinical practise.

\section{G218(P) A REVIEW AND AUDIT OF PENETRATIVE AND FORENSIC CHILD SEXUAL ABUSE CASES}

doi:10.1136/archdischild-2013-304107.230

${ }^{1} \mathrm{M}$ Cutland, 'K Castle, ${ }^{1,2} \mathrm{~A}$ Thomas. 'Community Paediatrics, St James University Hospital, Leeds, UK; ${ }^{2}$ Community Paediatrics, Leeds Community Healthcare NHS Trust, Leeds, UK

Aims To review all penetrative child sexual abuse (CSA) cases presenting to a local unit over 12 months and audit against national guidelines for sexually transmitted infection (STI) screening and Forensic medical examination appropriateness and timing.

Methods This was a retrospective audit of all penetrative CSA cases including oral, anal and vaginal penetration presenting to the unit over 12 months from January 2010

The medical reports were reviewed for demographic data including age, gender and relationship to perpetrator and the local database was searched for any previous medical reports on the same children. The Hospital's results server was checked for any STI results relevant to the cases and the findings were audited against national guidelines for both STI screening and for forensic medical examination appropriateness and timing. 